\title{
LA PERSONA Y SU DIGNIDAD AL INICIO DE LA VIDA: EL CON- CEBIDO POR TÉCNICAS DE FERTILIZACIÓN IN VITRO EN CHILE $^{1}$
}

Paulina Ramos Vergara ${ }^{2}$, Ángela Arenas Massa², Manuel Santos Alcántara ${ }^{3}$

Resumen: El presente trabajo analiza algunos de los problemas que plantea en Chile el inicio de la vida del concebido por técnicas de fertilización in vitro. De allí surge la necesidad de normar la aplicación de esas técnicas con el fin de proteger la vida en cumplimiento del mandato constitucional. Es un estudio descriptivo e histórico a partir de la revisión de proyectos de ley chilenos que regulan la materia — directa o indirectamente - entre 1996 y 2013. Se concluye que es urgente regular un estatuto jurídico del concebido no nacido para una garantía integral efectiva de su vida.

Palabras clave: técnicas de fertilización in vitro, concebido no nacido, criopreservación

Personhood and dignity at the beginning of life: the conceived by in vitro fertilization in Chile

Abstract: The present study analyzes some of the problems encountered in Chile in the beginning of life of those conceived by in vitro fertilization. From these arises the need to norm the application of these techniques with the goal to protect life in compliance with constitutional mandate. This is a descriptive historical study grounded on the review of Chilean project laws regulating the issue — direct and indirectly — between 1996 and 2013. It is concluded that is urgent to regulate a juridical statue of the unborn conceived to guaranty the effective integrity of his/her life.

Key words: in vitro fertilization techniques, unborn conceived, cryopreservation

A pessoa e sua dignidade no início da vida: o concepto obtido por técnicas de fertilizaçáo in vitro no Chile

Resumo: O presente trabalho analisa alguns dos problemas que apresenta no Chile o início da vida do concepto obtido por técnicas de fertilização in vitro. Dai surge a necessidade de normalizar a aplicação dessas técnicas com a finalidade de proteger a vida em cumprimento do mandato constitucional. É um estudo descritivo e histórico a partir da revisão de projetos de lei chilenos que regulam a matéria — direta ou indiretamente — entre 1996 e 2013. Conclui-se que é urgente regulamentar um estatuto jurídico do concepto não nascido para uma garantia integral efetiva de sua vida.

Palavras-chave: técnicas de fertilização in vitro, concepto não nascido, crio-preservação

\footnotetext{
${ }^{1}$ La presente investigación forma parte del Proyecto interdisciplinario "Destino de los embriones producidos por fertilización in vitro: Nuevos descubrimientos científicos y sus implicancias éticas y jurídicas a la luz del Magisterio de la Iglesia”, y es financiada por del Fondo de Investigación de la Vicerrectoría de Desarrollo de la Pontificia Universidad Católica de Chile, Dirección de Pastoral y Cultura Cristiana, 2012-2013. Su identificación es \# VRI 573/DPCC 2012.

${ }^{2}$ Centro de Bioética, Facultad de Medicina, Pontificia Universidad Católica de Chile, Chile

Correspondencia: pramosv@med.puc.cl

${ }^{3}$ Centro de Bioética, Facultad de Medicina, Facultad de Ciencias Biológicas, Pontificia Universidad Católica de Chile, Chile
} 


\section{Pichintu mongeliin peniewllelaiaiñ ${ }^{4}(1)$.}

\section{Introducción}

Hoy el hombre puede ser concebido en forma natural o por técnicas de reproducción asistida, entre ellas, la fertilización in vitro es una de las más complejas. El uso de biotecnología permite producir embriones in vitro, seleccionarlos, criopreservarlos, descongelarlos y transferirlos en el útero de una mujer. Ello muestra que el concebido por el uso de esas técnicas puede recibir una manipulación ilimitada(2). Al respecto se plantean, entre otras, las siguientes preguntas de orden jurídicofilosófico con repercusiones bioéticas: ¿cuáles son los principios que subyacen en el ordenamiento jurídico vigente en Chile respecto el inicio de la vida? ¿Cuáles son los intereses jurídicos involucrados? ¿Existe un deber de tutela jurídica hacia el concebido? ¿Cuáles son las posibilidades de acción respecto de los concebidos supernumerarios?

El uso de estas técnicas exige al Derecho y la filosofía respuestas efectivas; el reconocimiento de su dignidad permite considerar al embrión humano un ser existente, novedoso, alguien (sujeto) y no algo (objeto).

Las políticas de criopreservación, por otra parte, generan consentimientos informados diversos, incluso en el ámbito de los centros que se dedican a reproducción asistida(3); en algunos casos, parejas que consiguieron embarazo y nacimiento de un hijo posponen indefinidamente la transferencia embrionaria, o parejas titulares de muchos embriones en estado de criopreservación se encuentran con que estos poseen un mínimo potencial implantatorio (los concebidos más viables de la cohorte ya fueron transferidos) (4).

En este sentido, hay coincidencia en sostener que la criopreservación de los embriones detiene su proceso o actividad indefinidamente y se almacenan en espera de la implantación a un útero.

\footnotetext{
${ }^{4}$ La expresión aludida en mapudungún no tiene traducción literal al castellano. Su significado se refiere a la temporalidad; si es tan corto el tiempo de la existencia ¿no tendríamos que volver a vernos? Habla de la experiencia de amor humano y del reencuentro entre un hombre y una mujer. En materia de técnicas de reproducción asistida la reflexión del reencuentro/amor profundo de la pareja cobra una relevancia central y pareciera constituir la base de cualquier reflexión posterior.
}

A ello se suma que las capacidades de esa vida quedan privadas de un hábitat adecuado que le permitan realizarse y expresarse $(5,6)$; se priva al concebido, al menos temporalmente, de la acogida y de la gestación materna(7). Más aún, el destino de los embriones criopreservados puede ser su muerte y el empleo de sus tejidos para investigación biomédica.

En relación con la regulación jurídica internacional de estas técnicas, se observa la implementación de modelos orientativos(8) que dan cuenta del tratamiento al concebido por fecundación extracorpórea.

Con la finalidad de evaluar las diferentes legislaciones internacionales sobre la fertilización asistida en el contexto de los derechos del concebido, se realiza una búsqueda de los criterios orientadores de tales legislaciones con el objetivo de compararlas con las iniciativas legislativas chilenas y así orientar la próxima discusión nacional, ante la ausencia de una legislación específica y definitiva en Chile.

\section{Método}

Se trata de un estudio descriptivo e histórico. El método de conocimiento jurídico utilizado es sistemático, primero en el ámbito internacional y luego nacional(9). En el caso nacional, la muestra corresponde, en primer lugar, a fuentes normativas o proyectos de ley chilenos que, entre 1996 y 2013, proponen legislar — directa o indirectamente- las técnicas de reproducción asistida, y, en segundo lugar, artículos científicos y de referencia y fuentes iconográficas de actualidad.

\section{Resultados}

\section{Realidad internacional}

Los criterios orientadores de la regulación jurídica internacional se puede sintetizar en los siguientes:

- Países en que la legislación no regula las técnicas de reproducción asistida. El legislador y el Estado, mediante sus diversas manifestaciones, no intervienen, atendido a que la decisión de utilizar o no estas técnicas se consideran propias del ámbito privado de las personas; al Estado solo le corresponde garantizar el ejercicio de la autonomía individual. 
La consecuencia es que el concebido no implantado está en desprotección jurídica, puesto que no existe para él la posibilidad de expresar su autonomía. Su vida y desarrollo dependen del completo arbitrio y conveniencia de terceros, que no necesariamente son sus padres.

En estos países —algunos ejemplos son Chile, México(10) y Venezuela(11) — la regulación tiende a ser de carácter administrativo operativo, aplicable a los sistemas públicos de salud.

- Países en los que la legislación regula las técnicas de reproducción asistida, estableciendo límites mínimos en su práctica y efectos. En estos sistemas, la vida y tratamiento del concebido no implantado goza de relativa protección jurídica, en la medida en que se encuentra subordinado a las convenciones sociales que determinan las mayorías gobernantes. Entre estos, Reino Unido (1990)(12) y España $(2006)(13,14)$, que permiten técnicas de manipulación embrionaria, esto es, selección, experimentación, y criopreservación.

- Países en los que la legislación regula las técnicas de reproducción asistida, estableciendo límites en su práctica y prohibiendo efectos nocivos al concebido. El Derecho atiende a su razón de ser en cuanto responde a los valores propios del hombre y de su dignidad. Así, se reconoce su derecho a la vida y el trato respetuoso a la misma en todas las etapas del ciclo vital. Se invoca específicamente, en reproducción asistida, la Convención de los Derechos del Niño, particularmente el principio del interés superior del menor (en relación con el derecho a la identidad).

Entre estos países destacan Alemania (1990)(15), Austria (1992)(16), Suiza (1998)(17) e Italia (2004)(18). Sus ordenamientos jurídicos, entre otras normas, fijan un límite máximo de embriones que pueden obtenerse por ciclo (tres generalmente); disponen además que todos deben ser implantados a la madre, restringen la fecundación heteróloga y la criopreservación, y prohíben la selección genética, clonación y experimentación con embriones.

\section{Realidad nacional}

En sus inicios, las técnicas de reproducción asistida se ofrecían a las parejas estériles en subsidio a la procreación natural. Actualmente acceden a ellas las personas que cuentan con recursos económicos para ello, dada la limitada cobertura de las instituciones de salud previsional y Fondo $\mathrm{Na}$ cional de Salud(19).

En algunos de los centros sanitarios que realizan técnicas de reproducción asistida $(20)^{5}$ se criopreservan embriones(21) y se acumulan, puesto que sus padres, una vez que consiguen el embarazo y nacimiento - especialmente en partos múltiples-, posponen indefinidamente su transferencia; los embriones que habitan en este estado, se encuentran muchas veces con un mínimo potencial implantatorio (porque los más aptos de la cohorte ya fueron transferidos). Por otra parte, estudios recientes demuestran que los embriones con más días de cultivo in vitro tienen mejor tasa de implantación y, en consecuencia, la criopreservación disminuye la frecuencia de pacientes con embriones criopreservados y el número de embriones criopreservados(22).

Entre 1989 y 1995 nacieron en Chile 622 niños como resultados de procedimientos de reproducción asistida, incluyendo técnicas de donación de gametos(23). Entre 1990 y 2009 la Sociedad Chilena de Medicina Reproductiva registra 17.985 ciclo iniciados, 14.165 transferencias embrionarias, 5.543 infantes vivos gracias a las técnicas. Del total de infantes nacidos, 4.212 son producto de fecundación in vitro y fecundación in vitro con microinyección intracitoplasmática(24).

A la fecha, en Chile, cinco de los centros de reproducción asistida que funcionan son privados y dos están asociados al sistema público de salud. La seguridad, calidad y eficiencia de las técnicas de reproducción asistida en el país han experimen-

${ }^{5}$ Los centros de reproducción asistida acreditados por dicha entidad en Chile, son siete: Centro Diagnóstico Sanatorio Alemán (Concepción), CER -Centro Estudios Reproductivos (Santiago), Clínica de Mujer y Medicina Reproductiva (Viña del Mar), Programa Fertilización Asistida I.D.I.M.I. (Santiago), Unidad Medicina Reproductiva Clínica Alemana (Santiago), Unidad Medicina Reproductiva Clínica Las Condes (Santiago), Unidad de Medicina Reproductiva Monteblanco (Santiago. Clínica Las Nieves funcionó hasta el 2011; sus profesionales fundaron Monteblanco). También existe Fundación San Cristóbal. 
tado importantes avances, según datos publicados en la Revista Médica de Clínica Las Condes. Destacan que la tasa de parto con al menos un recién nacido vivo es mayor, a pesar del aumento de edad de la población sometida a ellas y de la disminución del número de embriones transferidos. Además, la frecuencia de multigestación extrema ha disminuido significativamente, debido a la criopreservación embrionaria (que permite transferencia secuencial) y a una fuerte campaña educacional.

\section{Tratamiento jurídico del concebido no nacido en Chile}

3.1 Constitución Política de la República de Chile (1980)(25)

El concebido no nacido es titular del derecho a la vida desde la concepción, según la Carta Fundamental. El derecho a la vida representa la facultad jurídica de exigir la conservación y protección de la actividad substancial propia del hombre en sus dimensiones física, biológica y también psíqui$\mathrm{ca}(26)$.

Se reconoce el valor de la vida en el artículo 19, $\mathrm{n}^{\circ} 1$ de la Constitución Política del Estado (y en los Tratados y Convenciones de Derechos Humanos ratificados por Chile), al asegurar a todas las personas el derecho a la vida. En su inciso $2^{\circ}$ prescribe que la "ley protege la vida del que está por nacer"; corresponde exclusivamente al legislador la función de determinar y aplicar la norma fundamental. Al respecto, consta en las actas de la Comisión de Estudio de la Nueva Constitución Política el acuerdo de garantizar por ley la vida del que está por nacer, desde su concepción, y el reconocimiento al concebido no nacido de su calidad de sujeto del derecho a la vida(27).

En igual sentido, la Convención Americana de Derechos Humanos, prescribe "persona es todo ser humano" (artículo 10) y "Toda persona tiene derecho a que se respete su vida. Este derecho estará por la ley y, en general, a partir del momento de la concepción. Nadie puede ser privado de ella arbitrariamente" (artículo $4^{\circ}$ inciso $\left.1^{\circ}\right)(28)$.

Todavía, la Convención sobre Derechos del Niño reconoce "el derecho intrínseco a la vida" (artícu- lo $1^{\mathrm{o}}$, no establece un límite mínimo para conceptualizar la niñez, solo el límite máximo de 18 años). Su preámbulo - que cumple una función interpretativa determinante- consigna: "el niño, por su falta de madurez física y mental, necesita protección y cuidado especiales, incluso la debida protección legal, tanto antes como después del nacimiento" (párrafo 9º)(29).

Con el fin de proteger la vida, en este caso de los concebidos no nacidos, se muestra en toda su importancia la necesidad de aplicar el principio "pro homine" (a favor de la persona) y "principio pro debilis" (favorecer a los débiles frente a los fuertes) en la interpretación y aplicación de las convenciones de derechos fundamentales(30).

3.2 Ley No 20.120 Sobre investigación científica en el ser humano, su genoma y prohíbe la clonación humana (2006)(31).

La discusión que da cuenta de la historia de la Ley No 20.120 es de gran valor, por cuanto en sede legislativa se discuten por primera vez temas de gran trascendencia para la vida y, en definitiva, para el género humano. Constituye la primera norma nacional de su jerarquía que hace referencia a la clonación humana; asume con criterio de realidad los avances tecnológicos que se están aplicando a los seres humanos.

En su artículo $1^{\circ}$ consigna sus fines, los que ciertamente son ambiciosos, dado que pretende garantizar la protección de la vida humana desde la concepción y la integridad física y psíquica de las personas; la diversidad e identidad genética humana en relación con la investigación científica biomédica y sus aplicaciones clínicas.

En su artículo $2^{\circ}$ reconoce el derecho o libertad de realizar investigaciones científicas biomédicas en seres humanos. Estas investigaciones deben garantizar los derechos y libertades esenciales de sus participantes.

En cuanto el respeto y garantía del derecho a la vida del concebido no nacido, hay disposiciones novedosas y de gran valor. A saber, se prohíbe toda práctica eugenésica, salvo la consejería genética (artículo 30), la práctica de la clonación en todas sus formas (artículo 50), la destrucción de 
embriones humanos para la obtención de células troncales que den origen a tejidos $\mathrm{u}$ órganos (artículo $6^{\circ}$ ). Es más, contempla sanción penal en caso de consejería genética y clonación, y presidio menor en su grado medio a máximo más inhabilitación del ejercicio de la profesión por el tiempo que dure la condena; en caso de reincidencia, la sanción será, además, la inhabilitación perpetua de ejercicio de la profesión (artículo 17). Se observa un vacío legal respecto de la pena por destrucción de embriones humanos para obtención de células troncales.

Esta ley es un avance de importancia hacia la protección del concebido no nacido. Se destaca que invoca la aplicación de la antigua institución romana del curator ventris o curator conceptis $(32)^{6}$ como criterio objetivo de responsabilidad; se concilian los derechos del qui in utero est $\mathrm{y}$ se tutela un interés público $(33,34)$; se protege indirectamente el spes nascendi del concebido criopreser$\operatorname{vado}(35,36)$.

\subsection{Resolución Exenta No 1072 Ministerio de Sa-} lud (28 de junio de 1985)(37,38)

La fertilización in vitro (FIV) y la transferencia embrionaria (TE) se encuentran reguladas por Resolución Exenta 1072, de 28 de junio de 1985 del Ministerio de Salud, que busca "orientar apropiadamente a los organismos afectos a la función normativa de este Ministerio y, por su intermedio, a la opinión pública y la comunidad..." (considerando tercero).

Dado que se trata solo de una Resolución Exenta del Ministerio de Salud, goza de limitada fuerza

\footnotetext{
${ }^{6}$ Constituye una institución clásica romana, que alude a un efecto previo al parto y consiste en el nombramiento de un custode durante el periodo que transcurre para el producto de la concepción, entre la fecundación y el nacimiento. Por custodia se entiende una actividad de vigilancia o guarda encaminada a la conservación de algo o alguien; custodia ventris tuvo en un primer momento una connotación patrimonial-sucesoria, pero origina los aforismos "qui in utero sunt in toto paene iure civili intellenguntur in rerum natura esse" (lo que están en el útero vienen considerados en totalidad por el derecho civil como si ya fuese acaecida su existencia legal) y "conceptus pro iam nato habetur" o regla de la ventaja del concebido (bizantina), y se ha extendido su aplicación a diversos ámbitos del Derecho. En bioderecho es oportuna la interpretación extensiva, porque tanto venter como conceptus, dicen relación al homo aún no nacido (alguien diferente de la madre). Venter, no es sinónimo del término castellano vientre, es decir no es portio de la madre; por ende el curator ventris es en realidad un curator concepti.
}

prescriptiva, restringida incluso al ámbito público; más bien, son normas orientadoras(39) y, en el ámbito privado, constituyen meras recomendaciones(40).

Del análisis de la Resolución Exenta se observa que declara su fundamento en el artículo $19 \mathrm{No}$ 1 de la Carta Política, el derecho a la vida. Comprende el derecho a procrear y, consecuentemente, el acceso a los medios médicos para superar las causas que impiden o dificultan. La administración con esta normativa expresa un nuevo derecho: "derecho a procrear".

Según esta Resolución Exenta se debe informar a la pareja solicitante de los riesgos de estos procedimientos y la necesidad de sus consentimientos; además dispone que la institución sanitaria y su Comité de Etica son responsables de revisar y aprobar el protocolo del procedimiento, registrar la descripción exacta de lo efectuado y el informe del mismo destinado a la pareja, dejando en claro los posibles riesgos existentes (numerando $7^{\circ}$ letra c, $9^{\circ}$ y $10^{\circ}$ complementan el punto).

También dispone que la institución sanitaria y el equipo de salud son responsables de proporcionar a la autoridad sanitaria los protocolos de trabajo en los que se registrarán los detalles del proceso de fecundación in vitro y transferencia embrionaria, indicando el número de huevos obtenidos, fertilizados o implantados. Y dispone que todos los óvulos fertilizados y normales deben ser transferidos a la madre, prohibiendo la criopreservación embrionaria para transferencia diferida de embriones, ni menos con fines de investigación" (artículo $8^{\circ}$ letra c) e inciso final).

3.4 Proyectos del ley sobre técnicas de reproducción asistida y/o derechos reproductivos tramitados en Chile entre 1993 y 2013

Los seis proyectos de ley sobre técnicas de reproducción asistida y afines (salud reproductiva) emanados del Congreso Nacional entre 1993 y 2013, desde el punto de vista de los valores/derechos que buscan proteger, se pueden clasificar entre aquellos proyectos que priorizan la protección a la vida y dignidad del concebido, su derecho a la identidad, y aquellos que prefieren la protección de la mujer, su libertad o elección reproductiva, 
igualdad y no discriminación.

3.4.1. Proyectos que priorizan la protección jurídica del concebido

Se encuentra en primer lugar, el proyecto que "Regula los principios jurídicos y éticos de las técnicas de reproducción asistida y establece sanciones para los infractores de sus normas" (boletín No 1026-07 de 6 de julio de 1993, autor Sebastián Piñera, archivado el 21 agosto de 2008)(41). Constituye el primer proyecto de ley en Chile sobre la materia, y los principios que fundamentan la moción son la protección a la vida y dignidad humana, interés superior del niño, protección a la familia y al matrimonio (revitaliza y refuerza el aforismo clásico mater sempre certa est).

En cuanto a la identidad del hijo, busca garantizarla limitando el acceso a las técnicas de reproducción asistida a matrimonios estériles (dentro del derecho a la salud); por tanto, en caso de dudas sobre la paternidad o maternidad contempla la prueba biológica a través del $\mathrm{ADN}$.

También considera el principio de proporcionalidad y precaución (considerando 3 y 4), a favor del paciente y del ser humano fruto de la concepción; reconoce el ius generandi como personalísimo (se impide la cesión de gametos a cualquier título, para evitar se lleven a cabo reproducciones asistidas heterólogas).

En cuanto el consentimiento de los cónyuges solicitantes, prescribe que debe ser libre, consciente, solemne (autorización notarial) e informado (artículo 6).

Respecto de la manipulación embrionaria, contempla la prohibición de técnicas de reproducción asistida con fines de experimentación genética, utilización de embriones con fines distintos a la procreación, maternidad subrogada (gratuita u onerosa) y la criopreservación. También prohíbe la comercialización de embriones. Contempla también sanciones penales y administrativas en caso de contravención (artículos 21 y 22).

Un segundo proyecto, denominado "De reproducción humana asistida" (boletín No 4346-11del 18 julio de 2006, autor Mariano Ruiz Esquide, archivado el 20 agosto de 2008)(42), fundamenta la moción a través de los principios de protección de la vida extracorpórea, autonomía y pluralismo en sociedad laica (artículo 2).

Este proyecto considera la fertilización homóloga para preservar la identidad del hijo y, solo en forma subsidiaria ante la ausencia o inutilidad de gametos, autoriza la fecundación heteróloga. Además, se remite al artículo 182 del código civil (artículo 6 y 10), según el cual los padres de hijos nacidos mediante técnicas de reproducción son el hombre y la mujer que se someten a ellas.

Al igual que el proyecto precedente, se hace mención al consentimiento de la pareja tratada, el que debe ser previo, libre, expreso e informado, dado personalmente, escrito, completo (artículo 3). La información derivada del procedimiento se considera protegida como dato sensible (según lo dispuesto en la Ley No 19.628 sobre Protección de la Vida Privada, artículo 2, g)(43).

También este proyecto prohíbe el uso de técnicas de reproducción asistida con fines distintos al procreativo. Y se refiere expresamente a la criopreservación y destrucción de embriones, contemplando sanciones penales y administrativas; considera además la posibilidad de adopción de embriones criopreservados, de acuerdo con la Ley No 19.629, una vez transcurridos cinco años desde el estado abandono (artículo 9 letra b).

El tercer proyecto de ley que considera sujeto al concebido se titula "Sobre reproducción humana asistida" (boletín No 4573-11 del 3 octubre de 2006, autor Guido Girardi, archivado el 5 enero de 2009) (44). Los principios que fundamentan la moción son la protección del hijo desde la fecundación, los derechos y libertades reproductivas de la mujer y del varón que se someten a las técnicas de reproducción asistida, y el principio de no discriminación en el acceso por razones económicas (considerandos 5 y 8 ).

Respecto del derecho a la identidad del hijo, pueden acceder a las técnicas las "personas", hombre o mujer, con fines terapéuticos y reproductivos (artículos 4 y 5); omite referencia a la fecundación heteróloga u homóloga. 
El proyecto de ley prescribe que el consentimiento debe ser, libre, informado, seguro e igualitario y previo (artículo 3 y 7 ).

La manipulación embrionaria no está permitida; no obstante, se admite la criopreservación "u otras", en la medida en que sea necesario para el éxito procreativo (artículo 5 inciso 2). Para efecto sancionatorio, se remite al Libro X del Código Sanitario (artículo 8).

3.4.2. Proyectos que priorizan la protección de los derechos reproductivos

En primer lugar, se observa el proyecto de ley "Sobre derechos sexuales y reproductivos" (boletín No 2608 -11 del 19 octubre de 2000, autores Allende, Ascencio y otros, archivado por rechazo de Comisión de Salud el 28 octubre de 2008) (45). Los principios que fundamentan la moción son los de libertad y dignidad de la mujer, autodeterminación (tomar decisiones reproductivas de manera informada y segura) y la ciudadanía activa (igualdad, equidad y empoderamiento). El proyecto se inserta en un contexto más amplio, en el que las técnicas de reproducción asistida se insertan como derechos reproductivos de carácter positivo, es decir, el Estado debe garantizar el derecho de reproducción de un sujeto (mujer o varón) que lo solicite, en las condiciones que mejor le convengan(46).

El proyecto, además, reconoce que las personas tienen derecho a tomar decisiones libres respecto de la procreación (artículo 11) y que existe derecho a la información y educación (artículo 6); no habla de consentimiento directamente.

Un segundo proyecto de ley "Sobre salud y derechos sexuales y reproductivos" (boletín No 5933 -11 del 1 julio de 2008, autores: Accorsi, Lobos, Rossi y otros, archivado el 10 marzo de 2011). Los principios que fundamentan la moción son libertad (autonomía), igualdad, equidad, empoderamiento, reconocimiento de la diferencia y subjetividad; prescribe el derecho a la educación. Reconoce derecho de mujeres y hombres a recuperar la fertilidad (artículo 9), y ejercicio de la sexualidad independiente de la reproducción (artículo 14).
El proyecto prescribe que las personas tienen derecho a tomar decisiones procreativas de carácter libre, informado y responsable. Se reconoce a mujeres y varones la posibilidad de tratamiento adecuado cuando la procreación no se ha conseguido (artículo 12).

Finalmente, el tercer proyecto de ley "Modifica el Código Sanitario, en orden de ampliar la protección de la maternidad incorporando un nuevo sentido y alcance al concepto de salud reproductiva, reconociendo la existencia de los trastornos de fertilidad de cualquiera de los integrantes de una pareja y su incidencia en salud" (boletín No 6624-11 del 28 julio de 2009, autores Chahuán, De Urresti, Enríquez Ominami y otros, en estado vigente, primer trámite constitucional en la Cámara de Diputados).

Los principios que fundamentan la moción son igualdad, derecho a la paternidad y maternidad, libertad y derecho a procrear. Respecto de las pautas que dicte la autoridad sanitaria en materia de reproducción asistida, el proyecto prescribe que esta debe remitirse a los parámetros de la Organización Mundial de la Salud (artículo 15B). Lo anterior incluye, entonces, temas relevantes como el derecho a la identidad, el consentimiento informado, la manipulación de embriones y la criopreservación de los mismos.

\section{Discusión}

1. El uso de la biotecnología debe respetar la dignidad inalienable de toda persona humana desde la concepción. Por tanto, la factibilidad es insuficiente como criterio para el uso de las técnicas de reproducción asistida. Existe necesidad de reflexionar - a la luz del Derecho nacional — sobre prácticas de fertilización in vitro y sus consecuencias.

En este sentido, retomar categorías clásicas como conceptis o concebido parece ser pertinente para referir el inicio de la vida humana en el ordenamiento.

2. El Derecho puede o no proteger la vida y limitar la manipulación del concebido por técnicas de reproducción asistida. En los casos en que no regula dichas prácticas, por considerarlas del ám- 
bito privado, olvida su fin: la procura del Bien Común.

En el caso de Chile, estas técnicas se realizan hace más de dos décadas sin ningún tipo de control ni regulación legal, en circunstancias que plantean nuevos dilemas, al disociar maternidad biológica y con ello la filiación.

$\mathrm{Al}$ respecto, se estima que dichas técnicas deben ser reguladas por el legislador de acuerdo con el principio de realidad y en cumplimiento del mandato constitucional que protege la vida de toda persona desde la concepción. La falta de regulación deja al concebido no implantado en la más completa desprotección y desamparo. Además, produce inconsistencias en el sistema de protección de los derechos fundamentales, dado que se vulnera en su esencia el derecho la vida, presupuesto de los demás derechos. Por último, el uso de estas técnicas no puede constituirse como "método alternativo de reproducción humana”.

Los proyectos de ley que buscaban regular la fertilización in vitro y la transferencia embrionaria están archivados.

3. Los proyectos de ley revisados que tienden a proteger al concebido se fundamentan principalmente en el ius generandi, derecho a la vida del concebido, principio de precaución y proporcionalidad, e interés superior del niño.

En relación con el derecho a la identidad del concebido, dos proyectos aparecen pronunciando una postura de protección (boletín No 1026-07 del 6 de julio de 1993 y boletín No 4346-11 del 18 julio de 2006); el tercero de ellos indirectamente lo desprotege (boletín No 4573-11 del 3 octubre de 2006).

Los proyectos son concordantes con las disposiciones de la Ley No 20.120, en relación con la manipulación y experimentación embrionaria. No obstante, en un proyecto se permite la "congelación u otras" en caso de ser necesario para el éxito procreativo (boletín No 4573-11 del 3 octubre de 2006).

Solo un proyecto de ley se refiere a la posibilidad de adoptar embriones criopreservados (boletín
No 4346-11, artículo 9 letra b).

Cabe destacar que los tres proyectos de ley referidos contemplan la regulación del consentimiento informado.

4. Los proyectos de ley revisados que tienden a proteger los derechos reproductivos se fundamentan principalmente en la libertad procreativa, dignidad de la mujer, autodeterminación, ciudadanía activa, igualdad, equidad, derecho a la paternidad y maternidad, y derecho a procrear.

Dos proyectos no se refieren al consentimiento informado, sea porque lo omiten o porque se remiten a pautas internacionales no vinculantes para el ordenamiento chileno (boletín No 260811 del 19 octubre de 2000 y boletín No 6624-11 del 28 julio de 2009, respectivamente). Un proyecto de este grupo hace referencia sucinta al consentimiento informado (boletín No 5933-11 del 1 julio de 2008).

Los proyectos de este grupo no se refieren al concebido como titular de derechos.

5. El concebido en estado de criopreservación está en posibilidad de nacer y, por ende, es sujeto especialmente vulnerable de protección jurídica; el reconocimiento de su personalidad implica asumir que es especialmente titular de derechos exigibles al Estado y a las demás personas.

En este sentido, la criopreservación debe prohibirse. Con ella se violenta el normal curso de crecimiento/desarrollo del concebido y queda a disposición de deseos e intereses ajenos. Existe voluntad de poder que determina y decide sobre la vida humana débil; reduce al conceptis a un valor de uso con fecha de caducidad, a un simple material biomédico.

6. A la fecha, las técnicas que dicen relación con el inicio de la vida de miles de chilenos se encuentran regulada solo por la Resolución Exenta $\mathrm{N}^{\circ}$ 1072, de 28 junio de 1985, del Ministerio de Salud, de limitado ámbito de aplicación.

Esta Resolución además plantea numerosas interrogantes, entre otras: ¿qué significa óvulos fertilizados y normales? ¿Cuál es el destino de los embriones que no se pueden transferir por ser 
calificados anormales? ¿Se les deja morir o se los elimina? ¿Es un tipo de muerte clínica? Si se declaran, ¿es posible destinarlos a investigación?

7. Se espera que el legislador vele por la vida, salud e integridad del gestado no nacido desde su concepción; que vele por sus derechos, entre estos, su derecho a nacer, a una familia estable, a conocer su ascendencia genética (rechazando el anonimato por parte del donante de gametos).

8. Con el avance de las tecnologías, el científico ha logrado descifrar el "know how" de la especie humana, sin tener claro los beneficios y los desastres que con ello puede generar. De ahí la gran preocupación y necesidad de replantear los valores de la persona y de la organización social, y de una regulación jurídica al servicio de la persona y de su dignidad. No se puede permitir que personas humanas - y el embrión lo es — sean tratadas como "objetos" que se pueden producir a medida — "a la carta"-, seleccionar de acuerdo a ciertos estándares de calidad, desechar por "anormales" y criopreservar para disminuir costos de futuros procesos de implantación. El deseo de ser padre no puede justificar desechar hijos.

11. La necesidad de regular el uso de estas técnicas de reproducción asistida también busca garantizar el respeto de la persona en su etapa embrionaria, su identidad genética, la integridad de las generaciones futuras y las prácticas destinadas a la homogeneización genética que violan el derecho a la diversidad.

En conclusión, ante la ausencia de una regulación específica, es urgente regular un estatuto jurídico del concebido no nacido para una garantía integral efectiva de su vida. 
La persona y su dignidad al inicio de la vida - Paulina Ramos, Ángela Arenas, Manuel Santos

\section{Referencias}

1. Mora Z. Palabras mágicas para reencantar la tierra. Santiago de Chile: Grupo Editorial Norma; 2003: 127.

2. D’ Agostino F. Parole di bioetica, Torino: Giappichelli; 2004.

3. Zunino N, Jaque JM. 20 años de criopreservación de embriones. La Tercera, 2013, agosto 23. Disponible en http:// www.latercera.com/noticia/tendencias/2013/08/659-539244-9-20-anos-de-criopreservacion-de-embriones.shtml Consultado el 26-08-2013.

4. Schwarze JE, Balmaceda J, Pommer R. La criopreservación embrionaria en estadios mas avanzados de desarrollo disminuye la frecuencia de pacientes con embriones criopreservados y el número de embriones criopreservados. Revista Médica de Chile 2012; 140: 45-49.

5. Astaburuaga MA. ¿Es éticamente correcta la adopción de embriones humanos congelados? Santiago de Chile: Tesis para optar al grado de Magíster en Bioética, Pontificia Universidad Católica de Chile; 2012.

6. Zurriariaráin G. La dignidad del embrión humano congelado. Revista médica Universidad de Navarra 2007; 51(1): 30-32.

7. Cogregazione per la Dottrina della Fede. Instrucción Donum Vitae, I, 6; AAS 80 (1988): 84-85.

8. Palazzani L. Introduzione alla biogiuridica. Torino: Giappichelli; 2002.

9. Álvarez G. Curso de investigación jurídica. 2a ed. Santiago de Chile: Lexis Nexis; 2005.

10. Grupo de información en Reproducción Elegida (GIRE). Informe No 6, abril 2007 a enero 2013, 2013; 6 (6.3): 168 174. Disponible en: http://informe.gire.org.mx/caps/cap6.pdf Consultado el 07- 07-2014.

11. Ministerio Público, República Bolivariana de Venezuela. Temas de interés. Vientre subrogado: ¿maternidad legal o biológica?, del 7 julio de 2014. Disponible en: http://www.mp.gob.ve Consultado el 03-08-2014.

12. Reino Unido. Ley británica sobre fertilización humana y embriología. Reino Unido: 1990. Disponible en: http://biblio. juridicas.unam.mx/libros/5/2292/81.pdf Consultado el 03-08-2014.

13. Ley 14/2006, de 26 de mayo, sobre técnicas de reproducción humana asistida. Disponible en: http://www.boe.es/boe/ dias/2006/05/27/pdfs/A19947-19956.pdf Consultado el 10-01-2014.

14. Berrocal A. Análisis de la nueva Ley 14/2006, de 26 de mayo sobre técnicas de reproducción humana asistida. Una primera aproximación a su contenido. Revista de la Escuela de Medicina Legal 2007; 4: 40-70.

15. Gesetz zum Schutz von Embryonen (Embryonenschutzgesetz-ESchG), 1990. Disponible en: http://www.gesetze-iminternet.de/eschg/BJNR027460990.html Consultado el 10-01-2014.

16. Fortplanzungsmedizingesetz (FMedG) BGBl. No 275/1992 id F BGBl. I No 98/2001. Disponible en: http://homepage.univie.ac.at/elisabeth.holzleithner/Fortpflanzungsmedizingesetz.pdf Consultado el 08-11-2013.

17. Loi fédérale 810.11 du 18 décembre 1998 sur la procréation médicalement assistée (LPMA). Disponible en: http://www. admin.ch/opc/fr/classified-compilation/20001938/index.html Consultado el 10-01-2014.

18. Legge 19 febbraio 2004, $\mathrm{n}^{\circ}$ 40. Norme in materia di procreazione medicalmente assistita. Gazzetta Ufficiale $2004 ; 45$. Disponible en: http://www.parlamento.it/parlam/leggi/040401.htm Consultado el 10-01-2014.

19. Schwarze JE, Zegers F, Galdalmes V. Reproducción asistida en Latinoamérica y Chile. Revista Médica Clínica Las Condes 2010; 21(3): 451-456.

20. Red Latinoamericana de Reproducción Asistida (REDLARA). Registro Latinoamericano de Reproducción Asistida. Disponible en: http://www.redlara.com/aa espanhol/quem centros.asp Consultado el 01-12-2013.

21. Hernández N. La criopreservación en Chile: aspectos éticos y estatuto jurídico aplicable. Memoria para optar al grado de Licenciado en ciencias jurídicas y sociales, U. Austral de Chile. Valdivia: Universidad Austral de Chile; 2007.

22. Schwarze JE, Balmaceda J, Pommer R. La criopreservación embrionaria en estadios más avanzados de desarrollado disminuye la frecuencia de pacientes con embriones criopreservados y el número de embriones criopreservados. Revista Médica de Chile 2012; 140: 45-49.

23. Zegers F. Consideraciones médicas e implicancias ético legales de la reproducción asistida en Chile. Centro Interdisciplinario de Estudios en Bioética, Universidad de Chile. Disponible en: http://www.uchile.cl/portal/investigacion/ centro-interdisciplinario-de-estudios-en-bioetica/publicaciones/76970/consideraciones-e-implicancias-dela-reproduccion-asistida-en-chile Consultado el 2-2-2013.

24. Schwarze JE, Crosby J, Zegers F. 20 años de reproducción asistida en Chile. Sociedad Chilena de Medicina Reproductiva. Disponible en http://www.socmer.cl/registro-chileno-1990-2009.pdf Consultado el 6-11-2013.

25. Constitución Política República de Chile 1980. Santiago: Editorial Jurídica de Chile; 2013. 
26. Verdugo M, Pfeffer E, Nogueira H. Derecho Constitucional. Tomo I, Santiago: Editorial Jurídica de Chile; 1994.

27. Gumucio JC. Procreación asistida. Un análisis a la luz de la legislación chilena. Santiago de Chile: Editorial Jurídica Conosur; 1997.

28. Organización de Estados Americanos, OEA. Convención Americana de Derechos Humanos. Disponible en: http://www. oas.org/dil/esp/tratados B-32 Convencion Americana sobre Derechos Humanos.htm Consultado el 10$11-2013$

29. Organización de Naciones Unidas, ONU. Convención de los Derechos del Niño. Disponible en: http://www.unicef.cl/ unicef/index.php/Texto-Oficial-de-la-Convencion Consultado el 23-12-2013.

30. Nogueira H. Derechos fundamentales y garantías constitucionales, tomo I, 2a ed. Santiago de Chile: Centro de Estudios Constitucionales de Chile, Universidad de Talca; 2008.

31. Biblioteca del Congreso Nacional República de Chile. Historia de la Ley No20.120 sobre investigación cientifica en el ser humano, su genoma, y prohibe la clonación humana. Disponible en: http://www.leychile.cl/ Consultado el 15-082013.

32. Talamanca M. Istituzioni di diritto romano. Milano: Giufré Editore; 1990.

33. Henríquez I. La regla de la ventaja para el concebido en el Derecho Chileno. Santiago de Chile: Legal Pubishing Chile; 2012.

34. Serrano Vicente M. Custodiam praestare: la prestación de custodia en el Derecho Romano. Madrid: Editorial Tebar; 2006.

35. Baccari MP. Curator Ventris: Il concepito, la donna e la res publica tra storia e attualità. Torino: G.Giapichelli Editori; 2012.

36. Ravinoich R. La tutela de los embriones congelados en la República Argentina (2004-2006). Disponible en: www. edictum.com.ar/.../Ricardo\%20D.\%20Rabinovich-Berkman.doc Consultado el 28-04-2013.

37. Centro de Bioética, Universidad del Desarrollo. Criopreservación de embriones humanos. Documento de trabajo. Disponible en: http://medicina.udd.cl/centro-bioetica/files/2010/10/Criopreservación.pdf Consultado el 5-122013.

38. Gobierno de Chile, Ministerio de Salud. Directorio Transparencia Activa. Disponible en: http://www.gobiernotransparentechile.cl/directorio/entidad/15/242/normativa a7c? $\mathrm{x}=0$ \&y=0\&page number=1 \&sort=tipo de norma\&direction=desc Consultado el 20-05-2013.

39. Corral H. Admisibilidad jurídica de las técnicas de reproducción artificial. Revista Chilena de Derecho 1992; 19: 439460.

40. Gumucio J. Procreación asistida: un análisis a la luz de la legislación chilena. Santiago de Chile: Editorial Jurídica Consur; 1997.

41. Congreso Abierto del Ciudadano Inteligente. Regula los principios jurídicos y éticos de las técnicas de reproducción asistida y establece sanciones para los infractores de sus normas. Boletín No 1026-07 del 6 de julio de 1993. Disponible en http:// congresoabierto.cl/proyectos/1026-07 Consultado el 3-09-2014.

42. Congreso Abierto del Ciudadano Inteligente. De reproducción humana asistida. Boletín No 4346-11 del 18 julio de 2006. Disponible en: http://congresoabierto.cl/proyectos/4346-11 Consultado en 10-04-2013.

43. Biblioteca del Congreso Nacional de Chile. Ley No19.628 sobre Protección de la vida privada. Disponible en: http:// www.leychile.cl/Navegar?idNorma=141599 Consultado en 2-12-2013-

44. Biblioteca del Congreso Nacional de Chile. Senado. Regula la aplicación de técnicas de reproducción humana asistida. Boletín 4573-11. Disponible en: http://www.senado.cl/appsenado/templates/tramitacion/index.php?boletin ini=4573-11 Consultado el 10-11-2013.

45. BibliotecadelCongreso NacionaldeChile. CámaradeDiputados. Estableceleymarcosobrederechossexualesyreproductivos. Boletín:2608-11. Disponible en: http://www.camara.cl/pley/pley detalle.aspx?prmID=1521\&prmBL=2608-11 Consultado el 2-12-2013.

46. Dalla Torre G, D’ Agostino F (ed.) La cittadinanza. Problemi e dinamiche in una società pluralistica. Torino: G. Giappichelli Editori; 2000.

Recibido: 5 de abril de 2014

Aceptado: 8 de julio de 2014 